\title{
Tecnologias da Informação e Comunicação, Função Composta e Regra da Cadeia
}

\author{
BARBOSA, S. M., Tecnologias da Informação e Comunicação, Função \\ Composta e Regra da Cadeia. 2009. 199f. Tese (Doutorado em Educação \\ Matemática) - Instituto de Geociências e Ciências Exatas, Universidade Estadual \\ Paulista (UNESP), Rio Claro, 2009. Tese orientada por Marcelo de Carvalho \\ Borba.
}

Débora da Silva Soares* Jhony Alexander Villa-Ochoa**

Esta resenha traz uma síntese do trabalho de pesquisa realizado por Barbosa sobre o ensino de Funções Compostas e Regra da Cadeia na disciplina de Cálculo Diferencial e Integral de uma variável. A inquietação da autora reside na dificuldade apresentada pelos estudantes no entendimento destes conteúdos. Sua proposta é desenvolver uma abordagem gráfica para estes conceitos, utilizando as Tecnologias da Informação e Comunicação (TIC) como recurso para a visualização e experimentação de conjeturas pelos alunos.

No primeiro capítulo, Barbosa identifica algumas das dificuldades que os alunos apresentam com relação ao conteúdo Regra da Cadeia, baseando-se na confrontação entre pesquisas realizadas por alguns autores e, também, em sua experiência como professora de Cálculo Diferencial e Integral I. Nessa confrontação ela visualiza que muitos erros cometidos por estudantes na compreensão da regra da cadeia estão enraizados em problemas com a

\footnotetext{
* Mestre em Matemática Pura pela Universidade Federal do Rio Grande do Sul (UFRGS). Aluna do Programa de Pós-graduação em Educação Matemática (Doutorado) da Universidade Estadual Paulista (UNESP), campus Rio Claro, SP, Brasil; membro do GPIMEM (Grupo de Pesquisa em Informática Outras Mídias e Educação Matemática). Endereço para correspondência: Av. 24-A, 1515, Bela Vista, CEP: 13506-900. Rio Claro, SP, Brasil. E-mail: debbie_mat@yahoo.com.br.

*** Doutor em Educação (Matemática) pela Universidade de Antioquia (UdeA), Medellín, Colômbia. Professor da Universidade de Medellín (UdeM), Medellín, Colômbia. Endereço para correspondência: Cra 85, n 53B-80, Apto 443, Medellín, Colômbia.E-mail: javo@une.net.co.
} 
compreensão da função composta. A autora também descreve sua trajetória de pesquisa, identificando suas inquietações, e apresenta o objetivo de sua pesquisa e a pergunta diretriz: "Como o coletivo, formado por alunos-com-mídias, produz conhecimento acerca de Funções Compostas e Regra da Cadeia, a partir de uma abordagem gráfica?" (BARBOSA, 2009, p.15).

No segundo capítulo, são apresentadas algumas definições de Função Composta encontradas em livros de Ensino Médio e de CDI. Segundo estudos discutidos por Barbosa, o foco principal das definições nestes livros é o algébrico e o mecânico, sendo que, muitas vezes, as ideias principais sobre este conceito não são explicitadas. Observa que há uma especial ênfase em conjuntos representados através do uso de diagramas, e afirma, ainda, que nem todas essas maneiras de apresentar a função composta enfatizam as restrições de seu domínio, assinalando, embasada na revisão da literatura, que, muitas vezes, isso é ignorado na sala de aula. Barbosa enfatiza que (a) o trabalho com composição de funções precisa de uma noção de função além da noção de processo que transforma elementos de um conjunto em elementos de outro conjunto e (b) uma abordagem gráfica, como apresentada por Husch (19952001), permitiria interpretar algumas características da composição de duas funções, por exemplo, as restrições nos domínios, permitindo, assim, a elaboração de conjeturas. A autora apresenta uma visão crítica sobre as vantagens e restrições apresentadas por algumas aproximações à compreensão da composição de funções, exemplificando, com o uso da linguagem coloquial, a ideia de decomposição de e as maneiras para expressar uma função como composição, e a desalgebrização, usando transformações como a translação e contração de gráfico. Em seguida, descreve o processo de obtenção do gráfico de uma função composta a partir dos gráficos de suas componentes, apresentado no site Visual Calculus I Composition of Function ${ }^{1}$, que foi base para a elaboração de suas atividades.

Dando continuidade a este capítulo, Barbosa faz uma discussão sobre a definição de derivada e, também, sobre como a Regra da Cadeia é apresentada em diferentes livros de CDI. De forma simplificada, a regra da cadeia explicita o modo de calcular a derivada de uma função que seja formada pela composição de outras duas funções deriváveis ${ }^{2}$. Segundo a autora, em geral a abordagem

\footnotetext{
${ }^{1}$ http://archives.math.utk.edu/visual.calculus/0/compositions.6/index.html (HUSCH, 1995-2001, p.14).

2 "Teorema: A Regra da Cadeia: Sejam $y=h(x)$ uma função derivável em $x$ e $x=g(t)$ uma função derivável em $t$. Consideremos a função composta $y=f(t)=h(g(t))$ com $\operatorname{Im}_{g} \subset D_{h}$. Então $f$ é derivável para todo $t \in D_{g}$ e $f^{\prime}(t)=h^{\prime}(g(t)) . g^{\prime}(t)$." (BARBOSA, 2009, p.42).
} 
das demonstrações e das definições é prioritariamente algébrica, o que poderia ser uma das fontes de dificuldades dos alunos com relação a este conteúdo. Além disso, a dificuldade de operar com a função composta também seria um fator determinante para esta situação.

O terceiro capítulo está destinado à apresentação de um mosaico de pesquisas cujo foco de estudo é a discussão de questões relacionadas ao processo de ensino e aprendizagem de Cálculo com a presença das TIC. A autora inicia com um breve histórico de pesquisas relacionadas à Matemática Avançada e às tecnologias. Em seguida, apresenta um levantamento sobre pesquisas especificamente relacionadas com o ensino de conteúdos de Cálculo com o uso das TIC. Como a autora observa,

As pesquisas apresentadas [...] têm indicado que as relações entre os aspectos algébricos, gráficos e numéricos podem ser enfatizadas na produção e compreensão de conceitos e suas aplicações, sugerindo que o papel das habilidades algorítmicas seja deixado a cargo dessas TIC (BARBOSA, 2009, p.59).

Algumas concepções sobre visualização, propostas por diferentes autores, são discutidas posteriormente e são exploradas algumas noções de visualização como habilidade, linguagem e metodologia. Como habilidade, a visualização é utilizada no sentido de interpretar e entender a informação figural e a capacidade de conceitualizar e transladar relações abstratas e informações não figurais (representações) em termos visuais; como linguagem, a visualização pode comunicar a Matemática, quando a abordagem algébrica não consegue ser expressa; como metodologia, a visualização possibilita a criação de conjeturas que podem ajudar no desenvolvimento matemático. Além disso, outras noções de visualização são apresentadas no texto, como "a habilidade, o processo e o produto de criação, interpretação, e o uso da reflexão" (ARCAVI, 2003, p.217) e como um processo natural que surge da riqueza das relações visuais existentes nos conceitos matemáticos, nas ideias e nos métodos, assim como no processo de transmissão e comunicação próprio da atividade matemática (GUZMÁN, 2002). Apesar de ser um aspecto muito importante da vida humana, o uso de imagens e a visualização ainda não têm um papel reconhecido na prova formal em matemática. A autora salienta esta importância da visualização, que está presente na concepção de diferentes autores, e afirma que as TIC trouxeram um novo impulso para o uso de imagens, devido à sua dinamicidade. 
Baseada nas ideias anteriores, Barbosa propõe sua própria interpretação da visualização como "um processo que associa a compreensão dos estudantes, entre si, e a mídia externa", indicando, ainda, que "é um processo essencial na elaboração de novas conjecturas que podem ser refutadas ou confirmadas" (BARBOSA, 2009, p. 62) e, partindo desta asserção, defende as representações múltiplas, destacando a importância do deslocamento entre as diferentes representações de um mesmo conceito para sua melhor compreensão.

Barbosa observa no trabalho de Allevato (2007) uma maneira de argumentar que as representações múltiplas são um recurso para a verificação de algumas conjeturas, feitas sobre determinados objetos matemáticos ou sobre a compreensão dos conceitos matemáticos. Apoia-se em Villarreal (1999) e Borba e Scheffer (2004) para ressaltar a importância de trabalhar com representações múltiplas, tanto na produção de conhecimentos quanto na atribuição de novos significados aos conteúdos por meio das interações com o computador.

O quarto capítulo é dedicado à discussão e análise sobre a produção de conhecimento e sobre o construto teórico seres-humanos-com-mídias. Baseandose em Moran (2006) a autora analisa o que seria conhecimento, apresentando a noção de construção de conhecimento como resultado de um processo de interação, de comunicação, considerando que conhecer é relacionar, integrar, contextualizar e fazer nosso o que vem de fora: "Conhecer é ir além da superfície, do previsível, da exterioridade, aprofundando os níveis de descoberta" (BARBOSA, 2009, p.70). Em seguida, baseia-se em Steinbring (2005) para analisar o que seria o conhecimento matemático. Contrariando a vertente que entende a Matemática como um objeto ideal, já existente e pronto, este autor afirma que o conhecimento matemático é construído através de atividades sociais e interpretações individuais, dando um caráter mais social à matemática. As declarações de Steinbring (2005) sobre a necessidade de fazer uma interpretação interativa dos conceitos e das notações matemáticas inspiram a autora desta tese a relacioná-las com as representações múltiplas e, no caso específico de funções, a autora acredita que podem ser potencializadas por um ambiente escolar em que os alunos e professores utilizam as TIC. Além disso, relaciona a produção de conhecimento matemático com o construto teórico seres-humanos-commídias de Borba e Villarreal (2005).

Este construto procura expressar a mútua influência que humanos e tecnologias exercem, um sobre o outro, na produção de conhecimento, e, também, evidenciar que a tecnologia é um ator neste processo. Mais ainda, a produção 
do conhecimento ocorre a partir da noção de um coletivo pensante sereshumanos-com-mídias, significando que os humanos são constituídos por tecnologias que transformam e modificam seu raciocínio e, ao mesmo tempo, esses humanos são constantemente transformados por essas tecnologias. Partindo dessa perspectiva, Barbosa assinala que Borba e Villarreal entendem que a visão dicotômica entre seres humanos e tecnologias não faz sentido.

Baseada nos elementos anteriores, essa pesquisadora explicita sua visão de conhecimento como uma produção própria (e diferenciada) de cada indivíduo, em um coletivo formado não só por humanos, como também por todas as representações e interfaces permeadas pela informatização.

No capítulo de número cinco, a autora justifica por que a abordagem de pesquisa se insere em um paradigma qualitativo e apresenta uma descrição dos procedimentos utilizados para a coleta de dados. Barbosa realizou projetos-piloto com estudantes de graduação em matemática e também um minicurso sobre o software Winplot, antes da coleta de dados, o que possibilitou repensar e melhorar as atividades com as quais iria trabalhar. A coleta de dados baseou-se na realização de experimentos de ensino, entendidos como um procedimento metodológico de coleta de dados que consiste em uma série de encontros entre os estudantes e o pesquisador, por um determinado período de tempo. Estes encontros foram realizados com cinco duplas de alunos do primeiro ano do curso de Licenciatura em Matemática, que fizeram o minicurso oferecido anteriormente pela autora. Os dados foram registrados com o auxílio do software Camtasia Studio, de uma filmadora fixa e de registros escritos realizados pela pesquisadora.

O capítulo sexto dedica-se à apresentação do perfil de cada uma das duplas de alunos que participou da coleta de dados, e a uma descrição dos episódios selecionados pela autora que continham indícios para responder a pergunta diretriz. Da descrição dos dados e de uma primeira análise emergiram algumas considerações: a interação com o software possibilitou a elaboração de conjeturas; a visualização foi importante para a comprovação de algumas destas conjeturas; houve a integração entre as representações gráficas e numéricas, sendo que isto foi possível devido à animação do software; o uso apenas da abordagem algébrica pode culminar em uma manipulação sem sentido de símbolos.

Além disso, a autora também constatou que os alunos: identificaram algumas propriedades para a composição de funções e da regra da cadeia; inferiram, mediante estratégias indutivas, a definição de regra da cadeia; identificaram algumas relações entre algumas funções não polinomiais e suas 
derivadas ainda desconhecidas por eles; mudaram a percepção sobre a importância do software. A pesquisadora ainda observou, por parte de alguns alunos, resistência ao uso de gráficos, além da preferência pela álgebra. Segundo a autora, isso pode estar sustentado em uma perspectiva formalista da matemática, que tem trazido consequências para o ambiente de ensino e aprendizagem da Matemática, levando os professores e, consequentemente, os alunos a pensarem em uma supremacia da álgebra em detrimento das imagens e dos gráficos. Nesse sentido, Barbosa (2009) discute a necessidade de oferecer a abordagem gráfica com um status semelhante aquele dado à álgebra.

No capítulo seguinte, a autora faz um aprofundamento da análise dos episódios descritos anteriormente, a partir da literatura estudada. Baseando-se em autores como Guzmán, Pierce e Stacey, Allevato, Steinbring, Borba e Villarreal e Lévy discute os seguintes temas: visualização, representações múltiplas e produção de conhecimento matemático como um processo coletivo. Sobre a visualização, a autora evidencia o seu papel fundamental na elaboração e desenvolvimento das atividades, além de pontuar sua presença natural nos processos humanos, incluindo aqueles relacionados com a produção matemática. Nessa pesquisa, o processo de visualização implícito na pergunta foi potencializado pelas Tecnologias da Informação e Comunicação (TIC) que, segundo a autora, transformam o modo como o conhecimento é produzido, reorganizando a forma de interagir e pensar. Baseado nisso, o conhecimento foi produzido através das discussões ocorridas entre os estudantes e em um processo de interpretação individual, expresso na forma oral, na forma escrita, ou na ação de trabalhar com o computador. Para a pesquisadora, esse processo individual não significa um indivíduo sozinho, mas imbricado de todo um coletivo que pensa junto com ele.

Com relação às representações múltiplas a autora salienta os momentos de coordenação entre elas ocorridos nos episódios, mostrando a importância deste movimento para a confirmação ou refutação de conjeturas, e seu papel de auxiliar alunos com dificuldades em representações algébricas. A questão relacionada com a produção de conhecimento matemático em um processo coletivo é discutida pela autora, e exemplos extraídos dos episódios sustentam que este processo inclui as mídias utilizadas pelos alunos, em particular, o software Winplot.

Finalizando, Barbosa reflete sobre a elaboração de sua tese e tece alguns comentários relacionados com a postura de um professor-pesquisador, destacando que seu trabalho traz elementos que podem auxiliar outros professores a 
elaborarem encaminhamentos diferenciados para o trabalho com os conteúdos de função composta e regra da cadeia. Sem o pressuposto de ser uma receita, as atividades elaboradas mostram a existência da possibilidade de uma abordagem envolvendo as TIC e a visualização para estes conteúdos. A autora mostra que a produção de conhecimento ocorreu por meio de elaborações realizadas pelos estudantes, através de conjeturas formuladas durante o processo de visualização potencializado pelas TIC. Segundo a pesquisadora, as "conjecturas foram confirmadas ou refutadas, levando-se em conta o entrelaçamento das representações múltiplas, que permearam todas as atividades, e por um coletivo pensante seres-humanos-com-mídias, no qual o ser humano transforma e é transformado pelas mídias em um processo interativo" (BARBOSA, 2009, p. 173). Com essas interpretações, a autora oferece uma resposta à sua pergunta de pesquisa.

A tese de Barbosa está bem estruturada quanto à organização dos capítulos e quanto às discussões elaboradas. Autores nacionais e internacionais, cujos trabalhos são relevantes, fizeram parte do referencial teórico, e a autora conseguiu articular de forma clara as considerações destes autores entre si e com suas próprias perspectivas com relação ao tema de estudo, fornecendo ao leitor um panorama coerente sobre as questões que permeavam o seu estudo.

Um primeiro comentário sobre o trabalho diz respeito aos dados colhidos pela autora: apesar de estarem registrados apenas os episódios que forneceriam algum indício para responder a pergunta inicial da pesquisa, percebe-se a riqueza de informações trazidas por eles. Sendo assim, outras questões são passíveis de ser abordadas a partir da sua análise. Por exemplo, uma análise mais aprofundada sobre as diferentes estratégias de resolução das atividades pelos alunos, as diferentes concepções dos alunos sobre os conceitos matemáticos trabalhados durante as atividades, e até mesmo a análise do discurso dos alunos ao expressarem seus argumentos. Outros questionamentos que surgem a partir da leitura da tese, e que poderiam gerar novas pesquisas, são: (a) a elaboração de uma reflexão sobre as potencialidades e limitações de cada representação, pois, como argumenta Villa (2001), tanto as representações gráficas quanto as tabulares contém limitações no reconhecimento de funções, particularmente as polinomiais. Assim, é possível discutir a necessidade de outorgar à abordagem gráfica um status semelhante ao do algébrico envolvido; (b) apoiando-se na noção de seres-humanos-com-mídias, como um coletivo pode avançar de uma compreensão da função composta como o resultado de uma operação a uma interpretação dela como uma nova função com suas próprias propriedades?; (c) 
como outros procedimentos metodológicos poderiam fornecer mais dados que permitam dar conta da produção de conhecimento através de uma negociação de significados entre os diferentes sujeitos?

A proposta da autora de desenvolver atividades com uma abordagem envolvendo a visualização e as TIC, para o tema em estudo, configura-se como uma possibilidade de trazer para o Ensino Superior oportunidades de transformação do modo tradicional de ensino, principalmente por conta das atividades propostas mostrarem caráter investigativo. Este tipo de trabalho já é bastante sugerido na escola básica. Porém, como pontuado por Barton (2008), é intrigante que atividades envolvendo jogos e investigações sejam removidas das salas de aula de nível secundário e superior.

De forma geral, a pesquisa de Barbosa incita à reflexão sobre diversos aspectos relacionados com o ensino dos conteúdos de Função Composta e Regra da Cadeia em cursos de nível superior, apresentando uma abordagem diferente da tradicional, pautada na visualização e no uso de TIC. Esta tese é uma boa leitura, tanto para professores quanto pesquisadores. Sendo assim, consideramos que a pesquisa desenvolvida pela autora mostra-se de grande relevância no âmbito da Educação Matemática.

\section{Referências}

ALLEVATO, N. S. G. Aspectos emergentes da utilização do computador na educação matemática. In: FRANZONI, M.; ALLEVATO, N. S. G. (Orgs.) Reflexões sobre a formação de professores e o ensino de ciências e matemática. Campinas: Alínea, 2007. Cap.4, p.75- 96.

ARCAVI, A. The role of visual representations in the learning of mathematics. Education Studies in Mathematics, Dordrecht, v. 52, n. 3, p. 215 - 241, 2003.

BARTON, B. The Languages of Mathematics: Telling Mathematical Tales. New York: Springer, 2008. (Mathematics Education Library, 46).

BORBA, M. C.; SCHEFFER, N. F. Coordination of multiple representations and body awareness. Educational Studies in Mathematics, Dordrecht, v. 57, n. 3, 2004. Videopaper.

BORBA, M. C.; VILLARREAL, M. E. Humans-with-media and the reorganization of mathematical thinking: information and communication technologies, modeling, experimentation and visualization. New York: Springer, 2005. 232 p. (Mathematics Education Library, 39). 
GUZMÁN, M. The role of visualization in the teaching and learning of mathematical analysis. In: INTERNATIONAL CONFERENCE ON THE TEACHING OF MATHEMATICS AT THE UNDERGRADUATE LEVEL, 2., 2002, Hersonissos. Proceedings... Hersonissos: University of Crete, 2002. p.1 - 24. Disponível em: <http:/ /www.math.uoc.gr/ ictm2/>. Acesso em: 9 mai. 2007.

HUSCH, L. S. Visual calculus: composition of function. Knoxville: Mathematics Department, University of Tennessee, 1995-2001. Disponível em: <http:// archives.math.utk.edu/visual.calculus/0/compositions.6/index.html>. Acesso em: 20 maio 2010.

STEINBRING, H. The construction of new mathematical knowledge in classroom interaction: an epistemological perspective. Dordrecht: Springer, 2005. 236 p. (Mathematics Education Library, 38).

MORAN, J. M. Ensino e aprendizagem inovadores com tecnologias audiovisuais e telemáticas. In: MORAN, J. M.; MASETTO, M. T.; BEHRENS, M. A. Novas tecnologias e mediação pedagógica. 12. ed. Campinas: Papirus, 2006. Cap.1, p.11 - 65. 173 p. (Coleção Papirus Educação).

VILLARREAL, M. E. O pensamento matemático de estudantes universitários de cálculo e tecnologias informáticas. 1999. 402 f. Tese (Doutorado em Educação Matemática) - Instituto de Geociências e Ciências Exatas, Universidade Estadual Paulista, Rio Claro, 1999.

VILLA, J. A. Identificar funciones polinómicas: Una tarea no siempre realizable. EMA, v. 6, n. 3, p. 290 - 307. jul., 2001. 
